\title{
Análise do Comportamento nas Organizações: Dados quantitativos da produção brasileira
}

\section{Behavior Analysis in Organizations: Quantitative data of Brazilian production}

\section{Análisis del Comportamiento en las Organizaciones: Datos cuantitativos de la producción brasileña}

\section{Erasmo Henrique de Araújo Nascimento', Marcelo Henrique Oliveira Henklain ${ }^{2}$}

[1] [2] Universidade Federal de Roraima I Título abreviado: Revisões sistemáticas da literatura e metanálises I Endereço para correspondência: Marcelo Henrique Oliveira Henklain, Universidade Federal de Roraima, Av. Cap. Ene Garcês, 2413 - Aeroporto, Boa Vista - RR, 69310-000 I Email: marcelo.henklain@ufrr.br I doi. org/10.18761/PAC.2020.v11.n2.04

Resumo: Existem diversas pesquisas sobre Análise do Comportamento nas Organizações desenvolvidas no Brasil, mas esta subárea da Análise do Comportamento ainda carece de maior divulgação e ampliação da produção. Diante disso, o objetivo deste estudo foi realizar uma revisão de literatura sobre a produção analítico-comportamental brasileira nas organizações no período de 1970 a 2017. Foram consultadas as seguintes fontes de informação: (a) dissertações e teses: sites de programas de pós-graduação com orientadores e/ou linhas de pesquisa em Análise do Comportamento e a Biblioteca Digital Brasileira de Teses e Dissertações; (b) artigos: Portal de Periódicos da Capes; (c) ao final das consultas, o banco de dados foi complementado com trabalhos da Biblioteca em OBM. Os descritores utilizados e os procedimentos de seleção de trabalhos variaram a depender da base de dados adotada. Os resultados sugerem que a produção nacional tem crescido na última década, apesar dessa produção estar concentrada no sul e sudeste do país. Verificou-se também, a partir de uma análise das palavras mais frequentes, compatibilidade entre os trabalhos encontrados e conceitos que caracterizam a teoria e a atuação analítico-comportamental. Sugere-se que pesquisas futuras ampliem o presente exame incluindo capítulos de livro e trabalhos apresentados em eventos.

Palavras-chave: psicologia organizacional e do trabalho; análise do comportamento nas organizações; revisão de literatura 


\begin{abstract}
There are several studies on Behavior Analysis in Organizations developed in Brazil, but this subarea of Behavior Analysis still lacks further dissemination and expansion of production. Therefore, the objective of this study was to carry out a literature review of the Brazilian behavioral-analytic production in organizations from 1970 to 2017. The following sources of information were consulted: (a) dissertations and theses: postgraduate programs sites with guidelines and / or lines of research in Behavior Analysis and the Brazilian Digital Library of Theses and Dissertations; (b) articles: CAPES Portal of Periodicals; (c) at the end of the consultations, the database was complemented with works from the OBM Library. The descriptors used and the procedures for research selection varied depending on the database adopted. The results suggest that the national production has grown in the last decade, although it is concentrated in the south and southeast of the country. It was also verified, considering an analysis of the most frequent words, compatibility between the studies found and concepts that characterize the behavioral-analytic theory and professional acting. Future research is suggested to extend the present exam including book chapters and studies presented at events.
\end{abstract}

Keywords: organizational and work psychology; behavior analysis in organizations; literature review.

Resumen: Hay varias investigaciones sobre Análisis del Comportamiento en las Organizaciones desarrolladas en Brasil, pero esta subárea del Análisis del Comportamiento todavía carece de mayor divulgación y ampliación de la producción. El objetivo de este estudio fue realizar una revisión de literatura sobre la producción analítico-comportamental brasileña en las organizaciones en el período de 1970 a 2017. Fueron consultadas las siguientes fuentes de información: (a) disertaciones y tesis: sitios de programas de postgrado con orientadores y / o líneas de investigación en Análisis del Comportamiento y la Biblioteca Digital Brasileña de Tesis y Disertaciones; (b) artículos: Portal de Periódicos de la Capes; (c) al final de las consultas, la base de datos se ha complementado con trabajos de la Biblioteca en OBM. Los descriptores utilizados y los procedimientos de selección de trabajos variaron dependiendo de la base de datos adoptada. Los resultados sugieren que la producción nacional ha crecido en la última década, aunque se concentra en el sur y sureste del país. Se verificó también, a partir de un análisis de las palabras más frecuentes, compatibilidad entre los trabajos encontrados y conceptos que caracterizan la teoría y la actuación analítico-conductual. Se sugiere que investigaciones futuras amplíen el presente examen incluyendo capítulos de libro y trabajos presentados en eventos.

Palabras clave: psicología organizacional y laboral; análisis de la conducta en organizaciones; psicología organizacional; revisión de literatura 
Uma organização pode ser definida como um sistema de interações comportamentais apresentadas por diversos trabalhadores orientados para a produção recorrente de resultados significativos para e na sociedade na qual a organização está inserida (Gusso \& De Luca, 2017). Esses resultados significativos dizem respeito a serviços e produtos de que as pessoas necessitam, tais como serviços de abastecimento de água e energia, de segurança pública e saúde, educacionais, de venda de produtos alimentícios e de vestuário, entre outros. Por essas razões, afirma-se que as organizações são importantes e uma constante na vida em sociedade, conforme concluem Careli (2010) e Zanelli, Bastos e Rodrigues (2014).

Essa produção de resultados significativos nas organizações, conforme a definição proposta, é fruto do trabalho humano. Logo, o sucesso de uma organização depende de como se comportam os seus profissionais. Por esse motivo, é necessário avaliar se o modo como são planejadas as contingências de um ambiente de trabalho de fato promovem produtividade, saúde, relacionamentos éticos, bem estar, alcance dos objetivos organizacionais etc. (Kienen \& Wolf, 2002).

Surge, então, a questão sobre como gerenciar o comportamento humano nas organizações de modo eficaz. Uma abordagem teórica da Psicologia que tem se destacado por intervenções eficientes e eficazes nas organizações é a Análise do Comportamento (AC) (Daniels \& Bailey, 2014). Conforme explicam Miguel (2001) e Moreira (2005), a AC vem demonstrando e ampliando métodos de pesquisa e tecnologias para a intervenção com o objetivo de dar suporte à administração do comportamento humano nas organizações.

A partir desse conhecimento são conduzidas intervenções em organizações tendo como objetivos analisar, compreender e propor medidas que favoreçam a solução de problemas organizacionais e a melhoria da qualidade de vida dos trabalhadores por meio da modificação das contingências que interferem nos comportamentos profissionais (Austin, 2000; Borba, Ramos, \& Costa, 2017). Nessas intervenções, segundo Aureliano, Arima e Careli (2013), busca-se atuar com base na proposição de contingências de reforçamento positivo para promoção de comportamentos profissionais associados ao alcance dos objetivos da organização. Além da mudança de contingências, busca-se ensinar aos administradores princípios sobre gestão do comportamento para que eles sejam capazes de aplicar, sistematicamente, esse conhecimento, promovendo desempenhos que contribuam com os objetivos organizacionais sem, nesse processo, prejudicar os trabalhadores.

Ao considerar a importância das organizações na vida em sociedade, o papel central do comportamento humano para o sucesso organizacional e as contribuições (existentes e possíveis) analítico-comportamentais no que se refere a intervenções organizacionais, surge a questão sobre o que já foi produzido de conhecimento científico nessa interface entre AC e organizações. Quantificar e caracterizar essa produção são tarefas próprias de uma revisão de literatura, que tem um papel importante no estímulo e orientação de novas pesquisas, além de facilitar uma avaliação crítica do trabalho desenvolvido. Segundo Colepicolo (2014), revisões de literatura são necessárias também para ajudar na organização da produção científica e na identificação do que pode ser feito para estimular o seu avanço.

Muito desse conhecimento analítico-comportamental sobre intervenções nas organizações foi sistematizado sob nomes diversos, tais como Gestão do Comportamento em Organizações, Organizational Behavior Management (OBM), Performance Management (PM), Análise do Comportamento nas Organizações (ACO) etc., suscitando, inclusive, questões sobre em que medida o trabalho conduzido sob tais expressões é, de fato, analítico-comportamental (Careli, 2013; Moreira, 2005). No Brasil, tem sido bastante difundida no meio acadêmico a sigla OBM (Borba, Ramos, \& Costa, 2017). Um exemplo disso foi observado no I Encontro Brasileiro de Análise do Comportamento nas Organizações, realizado em 2015, no qual essa expressão foi muito empregada nos trabalhos publicados nos anais do evento. Algumas traduções para essa expressão em inglês já foram propostas, tais como Gerenciamento Comportamental de Organizações e Gestão do Comportamento Organizacional (ou em Organizações), embora as divergências em relação à melhor expressão ou tradução persistam (Careli, 2010). Destaca-se que internacionalmente, OBM parece ser a expressão 
mais difundida considerando o nome do principal periódico consultado por analistas do comportamento interessados na interface AC e organizações: Journal of Organizational Behavior Management (JOBM). Outra expressão usual no Brasil é Análise do Comportamento nas Organizações (ACO), conforme indica o nome do evento supracitado. Essa questão da nomenclatura é relevante porque pode dificultar a comunicação de uma comunidade científica e a condução de trabalhos de revisão de literatura, revisão sistemática de literatura, metanálises, entre outros estudos cuja função é avaliar o conjunto da produção científica. Neste estudo, foi adotada a expressão ACO por enfatizar a interface entre uma abordagem da Psicologia e um campo de atuação profissional, sem delimitar um tipo de atuação (e.g., gerir) ou um conjunto de procedimentos específicos propostos por um autor específico (e.g., PM adotado por Daniels \& Bailey, 2014).

Avaliar a produção científica brasileira sobre a interface entre $\mathrm{AC}$ e organizações atualmente é relevante por, pelo menos, três motivos. O primeiro é ético. Se o conhecimento analítico-comportamental pode contribuir com o desenvolvimento das organizações brasileiras, então ele deve ser identificado, estimulado, divulgado e ensinado para os profissionais que atuam na gestão de comportamento humano nas organizações. $\mathrm{O}$ segundo relaciona-se ao primeiro e diz respeito a uma oportunidade. Sabese que o Brasil se destaca como o maior centro de Análise do Comportamento depois dos Estados Unidos (Todorov \& Hanna, 2010), o que indica a existência de uma comunidade científica que pode contribuir na produção e aperfeiçoamento da aplicação da AC nas organizações. O terceiro motivo é que poucos trabalhos dedicaram-se a examinar a produção brasileira sobre ACO. Muitos trabalhos de revisão da literatura ou de metanálise conduzidos no Brasil avaliaram a produção publicada no JOBM (e.g., Moreira, 2005; Careli, 2013). Portanto, é por esses motivos que um estudo de revisão pode ser considerado necessário. Os parágrafos seguintes buscam apresentar o que já se sabe sobre a produção brasileira acerca da ACO.

Gusso e De Luca (2017) examinam a história da Análise do Comportamento nas Organizações (ACO) no Brasil contribuindo, assim, com a indicação de variáveis relevantes que influenciaram a produção científica na área, além de apresentarem informações que ajudam a identificar o que foi pesquisado ou realizado no Brasil em relação à ACO. Esse estudo é útil enquanto suporte para os analistas do comportamento desenvolverem novas pesquisas e intervenções. Os autores relatam que o estudo desenvolvido na década de 1970 por Sílvio Paulo Botomé, que reestruturou os serviços prestados pela Secretaria de Saúde do município de São Paulo, foi possivelmente a primeira intervenção utilizando os conceitos, princípios e procedimentos da AC em organizações e que foi amplamente divulgada no país. Além desse estudo, os autores destacaram as contribuições de Antônio Camilo, psicólogo que realizou diversas atividades relacionadas ao campo de recursos humanos na Eletropaulo, em São Paulo, utilizando AC em contextos organizacionais. Essas duas contribuições consistem, segundo o exame de Gusso e De Luca (2017), nos marcos brasileiros de início das experiências aplicadas do trabalho de analistas do comportamento em contextos organizacionais.

Deve-se considerar também, segundo Gusso e De Luca (2017), as intervenções na área de educação profissional. As contribuições decorrentes dos trabalhos com Ensino Programado, aperfeiçoados no Brasil a partir das contribuições de Carolina Bori e denominados de "Programação de Condições de Ensino" (Kubo \& Botomé, 2001) e, recentemente, como "Programação de Contingências para Desenvolver Comportamentos" (Kienen, Kubo, \& Botomé, 2013), estiveram presentes em experiências no CENAFOR (Centro Nacional de Formação Profissional) e no curso de especialização em Programação de Ensino desenvolvido na Universidade de São Carlos. Ressalta-se que tais estudos são intervenções educacionais orientadas para objetivos organizacionais ou para a promoção de comportamentos profissionais, existindo, portanto, uma interface com a atuação em organizações.

Outro ponto que merece destaque, segundo Gusso e diz respeito à formação de analistas do comportamento qualificados para atuar no campo organizacional. Nesse sentido, a primeira linha de pesquisa em $\mathrm{ACO}$ foi concebida em um programa de Pós-graduação no Brasil específica em Análise do Comportamento em Organizações, Trabalho e Aprendizagem na Universidade Federal de Santa 
Catarina. Essa linha de pesquisa iniciou em 2006 e encerrou suas atividades em $2013 \mathrm{com}$ a aposentadoria dos professores que a mantinham, José Gonçalves Medeiros, Olga Mitsue Kubo e Sílvio Paulo Botomé, tendo formado 27 mestres e 17 doutores.

Com relação à divulgação de conhecimento científico em eventos na área, ocorreu um aumento na disseminação da ACO. Segundo dados de Gusso e De Luca (2017), desde 2010 foi criado o Grupo de Interesse Especial (GIE) em OBM nos Encontros Anuais da Associação Brasileira de Psicologia e Medicina Comportamental (ABPMC). O seu objetivo era o de avaliar o desenvolvimento desse campo de atuação e propor encaminhamentos para o seu desenvolvimento e consolidação no Brasil. A partir deste GIE, foi realizado em 2015 o I Encontro de ACO e a criação da Biblioteca em OBM, que reúne textos sobre ACO elaborados por analistas do comportamento brasileiros, entre outros autores. Notase, então, um crescimento do interesse e da produção de analistas do comportamento em relação à Análise do Comportamento nas Organizações (Gusso, 2017; Gusso \& De Luca, 2017).

Uma lacuna identificada em relação à contribuição de Gusso e De Luca (2017) é que os autores não descreveram quantitativamente a produção analítico-comportamental brasileira nas organizações, o que auxiliaria a dimensionar o volume da produção nacional e o seu potencial de crescimento, bem como forneceria evidência empírica sobre quais são os principais laboratórios e orientadores. Essas informações são úteis para analistas do comportamento interessados em atuar na pesquisa e na prestação de serviços em relação às organizações, além de fornecer uma complementação do quadro traçado por Gusso e De Luca (2017), o que pode ajudar no registro histórico e na organização da produção científica da área.

Finalmente, destaca-se que a atuação em Psicologia Organizacional e do Trabalho no Brasil parece permanecer muito restrita a alguns poucos processos de atuação do psicólogo, muitas vezes conduzidos sem a devida fundamentação científica. Um exemplo dessa realidade pode ser nota- da em estudo conduzido por Gusso, Alvarenga, Nunes, Nunes, De Luca e Oliveira (2019), cujo objetivo foi descrever a atuação de psicólogos organizacionais na Região Sul do Brasil. Verificouse que $53 \%$ da amostra declarou trabalhar com processos seletivos e $55 \%$ com treinamento e desenvolvimento. Contudo, apenas $4 \%$ dos profissionais declarou realizar análise de cargos. Esse dado preocupa uma vez que analisar cargos é um pré-requisito para a realização de seleções e processos de capacitação.

Mesmo a avaliação de desempenho é pouco realizada, apenas $17 \%$ da amostra. Esse processo é um dos fundamentos para orientar o desenvolvimento humano nas organizações. Sem ele, a realização de capacitações pode estar sendo orientada por critérios pouco válidos. Nesse sentido, é preciso avaliar se a produção científica em ACO está sendo conduzida de modo a lidar com essa realidade, o que requer, por exemplo, uma produção diversificada, teoricamente consistente e metodologicamente rigorosa. No presente estudo, será possível avaliar apenas a primeira dimensão: grau de diversificação dos estudos.

No estudo de Gusso et al. (2019), verificou-se também que apenas $9,07 \%$ da amostra declarou orientar o trabalho desenvolvido em organizações com base na abordagem analítico-comportamental. É possível que a produção na área precise ser mais divulgada para que os profissionais possam se beneficiar das contribuições da ACO.

Tendo em vista o que foi apresentado, o objetivo deste estudo foi conduzir uma revisão da literatura com foco na descrição quantitativa, no período de 1970 a 2017, da produção científica brasileira relacionada à Análise do Comportamento nas Organizações (ACO). O período definido se inicia na década de 1970 em decorrência dos trabalhos de Sílvio Paulo Botomé e Antônio Camilo, considerados precursores das primeiras experiências aplicadas de AC em contextos organizacionais (Gusso \& De Luca, 2017); o término da pesquisa foi em 2017 porque foi o ano anterior a finalização deste estudo que consistiu no Trabalho de Conclusão de Curso do primeiro autor. 


\section{Método}

\section{Materiais}

Foi utilizado notebook com Sistema Operacional Windows $^{\oplus} 10$ e acesso à internet, bem como os softwares Microsoft Excel e Iramuteq (Ratinaud, 2009). Foram criadas duas planilhas eletrônicas no Excel para armazenar os dados deste estudo. As informações coletadas em cada trabalho selecionado em função dele ser uma "Dissertação ou Tese" foram: Tipo de trabalho; Nome do(s) autor(es); Título; Nível (especialização, mestrado ou doutorado); Ano de publicação; Instituição/programa a que o(s) autor(es) estava(m) vinculado(s) na época da pesquisa; Nome do orientador; Objetivo da pesquisa; Fonte na qual o trabalho foi encontrado; Link da fonte; Resumo. No caso do "Artigo" foram: Tipo de trabalho; Nome do(s) autor(es); Título; Ano de publicação; Instituição/programa a que o(s) autor(es) estava(m) vinculado(s) na época da pesquisa; Objetivo da pesquisa; Fonte na qual o trabalho foi encontrado; Link da fonte; Resumo. A base de dados completa está disponível no seguinte endereço: https://osf.io/pdjnq/ (doi: 10.17605/OSF.IO/PDJNQ).

\section{Fontes de obtenção dos estudos}

As fontes variaram em função do tipo de estudo. Ao final da consulta às fontes selecionadas, foi consultado também o Site da biblioteca virtual de publicações em OBM (http://bit.ly/2T7GMZm) para complementar o banco.

Dissertações e Teses. Esta consulta foi feita na Biblioteca Digital Brasileira de Teses e Dissertações (http://bdtd.ibict.br/) e em sites de programas de pós-graduação (PPG) de Análise do Comportamento ou com linhas de pesquisa em Análise do Comportamento. Os seguintes programas foram selecionados com base em Henklain, Carmo e Haydu (2017): (a) PPG de Psicologia da UFSCar; (b) PPG de Análise do Comportamento da UEL; (c) PPG de Psicologia Experimental: Análise do Comportamento da PUC-SP; (d) PPG de Teoria e Pesquisa do Comportamento da UFPA; (e) PPG de Ciências do Comportamento da UNB; (f) PPG em Psicologia da Universidade Federal de Santa Catarina (UFSC); (g) PPG em Psicologia Experimental da USP; (h) PPG em Psicologia da PUC-GO; (i) PPG em Psicologia UNESP-Bauru; e (j) PPG Instituto Paradigma São Paulo.

Artigos. Esta consulta foi conduzida no Portal de Periódicos da CAPES (http://www.periodicos.capes.gov.br).

\section{Procedimentos de coleta}

Critérios de seleção e de exclusão de estudos. A partir da consulta às fontes de informação, os estudos identificados foram selecionados com base nos seguintes critérios: (a) ter sido publicado dentro do período definido nesta pesquisa, isto é, 1970 a 2017; (b) ser uma produção nacional; e (c) ser um estudo sobre Análise do Comportamento nas Organizações. Foram considerados trabalhos da abordagem comportamental aqueles que (a) mencionaram no título ou no resumo tratar-se de um estudo analítico-comportamental ou (b) que apresentaram no resumo o objetivo de realizar uma análise comportamental ou (c) que empregaram no título ou no resumo conceitos analítico-comportamentais.

A cada estudo identificado lia-se o título e, sempre que necessário, o resumo para avaliar se ele deveria ser incluído no trabalho. Trabalhos duplicados foram removidos ao longo do processo de coleta de dados.

Descritores utilizados. $\mathrm{Na}$ BDBTD foi utilizado o descritor < "organizational behavior management" $>$ e no Portal de Periódicos da CAPES foi utilizado < "análise do comportamento" AND organizaç > Nos testes feitos antes da coleta, esses foram os descritores que, em cada base de dados, apresentaram os melhores resultados. A escolha de descritores foi baseada nas expressões mais comuns observadas nos trabalhos que compuseram a introdução deste estudo. No caso dos sites dos programas de Pósgraduação, todos os títulos dos trabalhos foram lidos e, quando necessário, os resumos também foram consultados. 
Procedimentos de análise de dados

Dados gerais. Quantos trabalhos já foram publicados no Brasil por tipo? Foi realizada a contagem da quantidade de trabalhos por tipo ("Dissertação e Tese" e "Artigo") publicados por ano e calculada a frequência acumulada para auxiliar na avaliação sobre em que medida a produção tem crescido, diminuído ou permanecido estagnada. Quais são as palavras mais frequentes nos estudos selecionados e como estão relacionadas? Foi criada uma nuvem de palavras a partir do software Iramuteq (Ratinaud, 2009). A nuvem de palavras tem como objetivo representar graficamente um conjunto de termos de acordo com as suas frequências em um determinado texto. Por ser uma análise que facilita a identificação de palavras-chave a partir do banco de dados (corpus textual), é utilizada em contextos nos quais seja relevante identificar os principais conceitos e termos presentes em um conjunto de informações textuais (Ratinaud, 2009). Quais foram os objetivos mais frequentes dos trabalhos? Foram criadas seis categorias: (a) revisão de literatura e metanálise; (b) análise conceitual, histórica e filosófica; (c) Análise funcional de comportamentos profissionais e descrição de contingências em contexto de trabalho; (d) Caracterização, Descrição e Decomposição de comportamentos profissionais; (e) Avaliação de eficácia e/ou eficiência de programa de ensino ou de intervenções de um modo geral; (f) Adaptação, construção e investigação de evidências psicométricas de instrumentos avaliativos e descritivos.

Dados específicos das Dissertações e Teses. Quem são os orientadores que mais orientaram pesquisas e a que instituições pertencem? Foi realizada a contagem do número de Teses e Dissertações por orientador e instituição.

Dados específicos dos Artigos. Quem são os autores que mais publicaram artigos? Foi contabilizado o número de trabalhos por autor.

\section{Resultados}

Foram identificados 26 estudos do tipo "dissertação e tese", dos quais 19 foram dissertações e sete foram teses. Na BDBTD foram selecionados apenas três estudos, nos sites dos programas de pós-graduação foram selecionados $18 \mathrm{e}$, por fim, cinco estudos foram incluídos após a consulta à Biblioteca de publicações em OBM. De artigos foram selecionados 19 estudos, sendo três deles oriundos do Portal de Periódicos da CAPES e 16 incluídos após acesso à Biblioteca de publicações em OBM. Ao todo, foram avaliados 45 estudos. Um aspecto importante é que a maioria dos estudos encontrados nas bases de dados selecionadas já haviam sido catalogados na Biblioteca de publicações em OBM, o que confirma a fidedignidade das informações divulgadas neste site, permitindo que futuras pesquisas utilizem-no como fonte de informações.

A Figura 1 exibe duas curvas de frequência acumulada, uma representando dissertações e teses publicadas no Brasil (linha preta com valores indicados por pontos) e a outra os artigos (linha cinza com valores indicados por quadrados). Nessa figura nota-se que o primeiro trabalho identificado foi em 1981 e até 2002 a produção nacional permaneceu estagnada. Só a partir de 2002 é que se observa uma aceleração positiva das curvas, indicando crescimento na produção. No período de 2006 a 2011 observa-se o maior grau de aceleração da curva de teses e dissertações. A partir desse ponto, essa curva cresce de modo estável com uma média de 1,5 trabalhos por ano. De 2012 a 2017 a aceleração positiva também foi elevada. Para a produção de artigos o período de 2011 a 2013 foi o mais intenso. Após esse período a produção estagnou um pouco, tendo apresentado média de 0,83 trabalhos por ano. É possível notar que de 1988 a 2009 a produção de artigos foi superior à de "dissertações e teses", ainda que a curva tenha uma aceleração suave, tendo permanecido sem alterações no período de 1990 a 2000. Os anos com maior número de teses e dissertações foram 2010 e 2011, ambos com três trabalhos. O ano com maior produção de artigos foi 2013, com três estudos publicados. 


\section{Teses, Dissertações e Artigos}

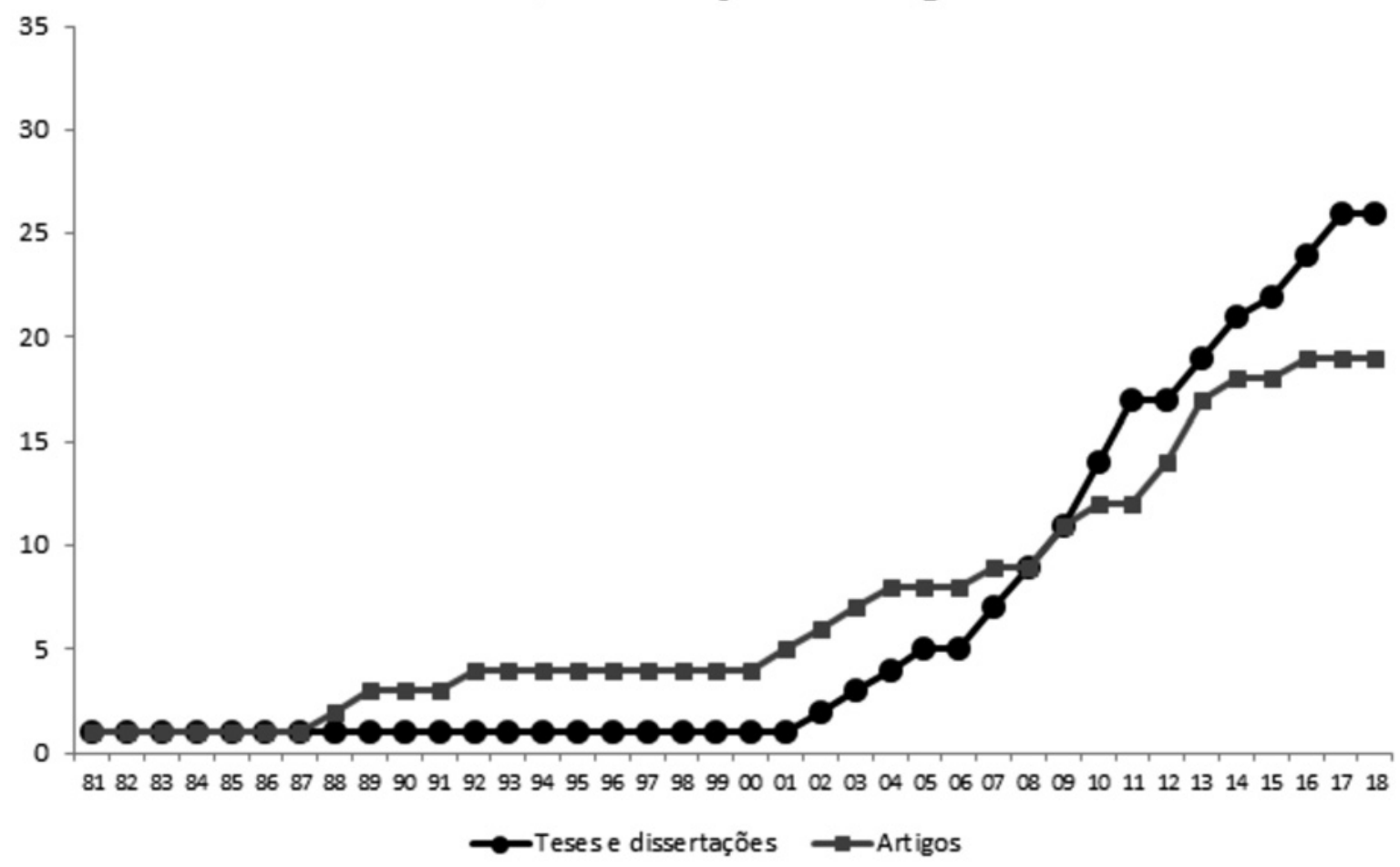

Figura 1. Teses, dissertações e artigos sobre Análise do Comportamento nas Organizações.

A seguir, na Figura 2, é apresentada a nuvem de palavras relativa às Teses, Dissertações e Artigos, que agrupa e organiza as palavras em função da sua frequência a partir dos resumos desses estudos (parte superior da figura) e dos descritores/palavras-chave (parte inferior da figura).

$\mathrm{Na}$ Figura 2, parte superior, é possível identificar os termos mais frequentes nos resumos. As maiores palavras são aquelas cujo frequência é superior em comparação com as demais, a saber: comportamento (162 ocorrências), organização (64 ocorrências), classe (60 ocorrências), trabalho (58 ocorrências), processo (58 ocorrências), estudo (56 ocorrências), profissional (52 ocorrências) e resultado (49 ocorrências). Dos 45 estudos, um dos trabalhos não tinha resumo ou palavras-chave, de modo que a nuvem foi construída sem ele. Ainda na Figura 2, parte inferior, dos 45 estudos, apenas 41 apresentaram descritores/palavras-chave. Notase que as palavras-chave de destaque foram: com- portamento (24 ocorrências), organizacional (15 ocorrências), ensino (10 ocorrências), trabalho (10 ocorrências), profissional (9 ocorrências), análise do comportamento ( 9 ocorrências), psicologia (8 ocorrências) e gestão (8 ocorrências).

A Tabela 1 exibe os objetivos mais frequentes em relação aos 45 estudos identificados (dissertações, teses e artigos). Nota-se uma concentração da produção de pesquisas em torno da "Caracterização, Descrição e Decomposição de comportamentos profissionais" (15 estudos; 33,33\%), da "Análise conceitual, histórica e filosófica" (10;22,22\%) e da "Análise funcional de comportamentos profissionais e descrição de contingências em contexto de trabalho" (9;20,00\%). Existe uma produção menor no que concerne à avaliação de eficiência de procedimentos e à construção de instrumentos (ambos com apenas três estudos cada).

A Tabela 2 exibe os nomes dos pesquisadores que mais orientaram estudos sobre ACO e as instituições com maior produção. 


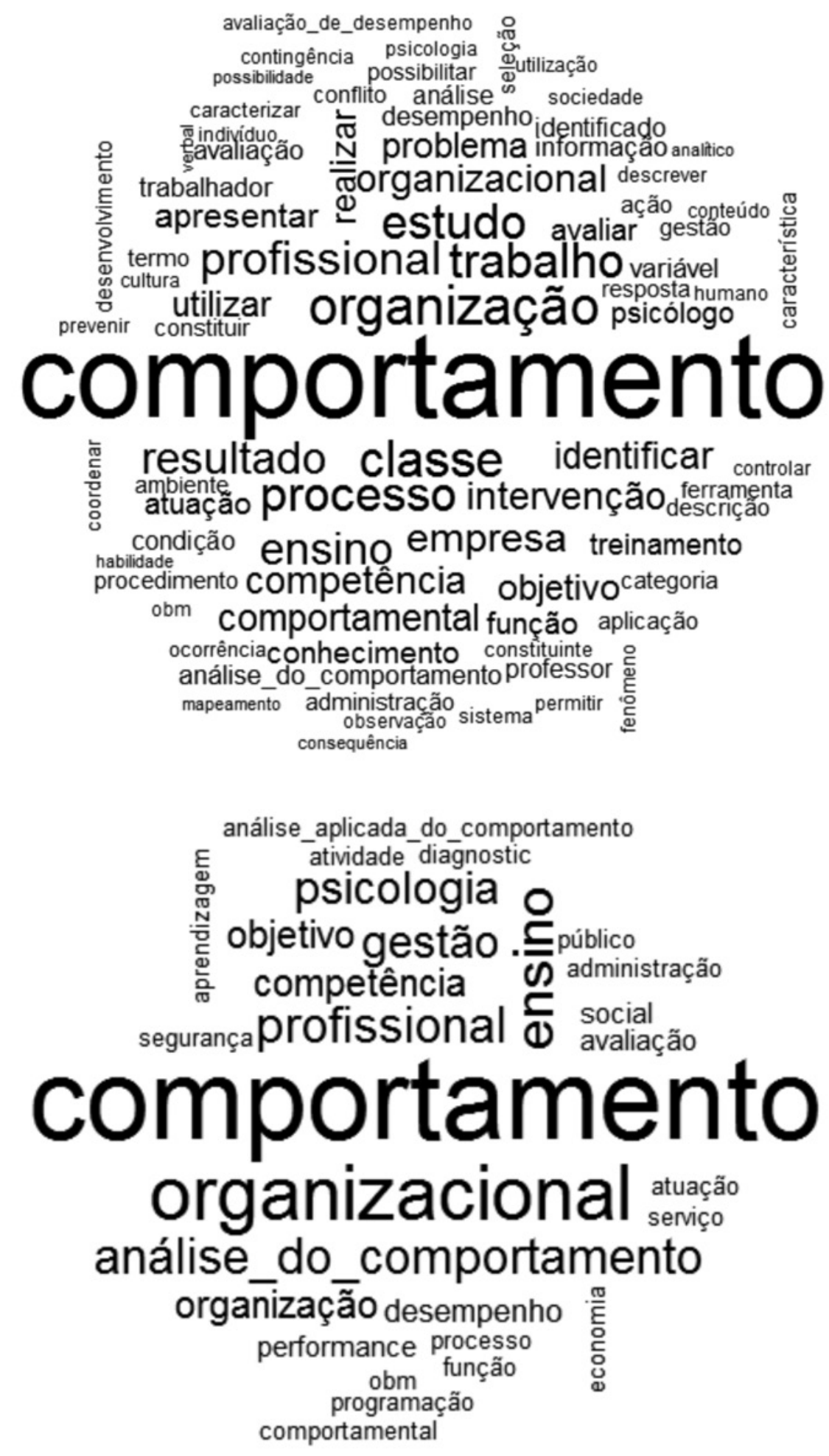

Figura 2. Nuvem de palavras construída a partir dos resumos das Teses, Dissertações e Artigos encontrados (parte superior) e Nuvem de palavras construída a partir dos descritores/palavras-chave das Teses, Dissertações e Artigos encontrados (parte inferior). 
Tabela 1. Quantidade e porcentagem de estudos (teses, dissertações e artigos) por tipo de objetivo.

\begin{tabular}{|c|c|c|}
\hline Categoria & Qtd. & $\%$ \\
\hline Caracterização, Descrição e Decomposição de comportamentos profissionais & 15 & 33,33 \\
\hline Análise conceitual, histórica e filosófica & 10 & 22,22 \\
\hline $\begin{array}{l}\text { Análise funcional de comportamentos profissionais e descrição de contingências } \\
\text { em contexto de trabalho }\end{array}$ & 9 & 20,00 \\
\hline Revisão de literatura e meta-análise & 5 & 11,11 \\
\hline $\begin{array}{l}\text { Avaliação de eficácia e/ou eficiência de programa de ensino ou de intervenções } \\
\text { de um modo geral }\end{array}$ & 3 & 6,67 \\
\hline $\begin{array}{l}\text { Adaptação, construção e investigação de evidências psicométricas de instrumen- } \\
\text { tos avaliativos e descritivos }\end{array}$ & 3 & 6,67 \\
\hline Total & 45 & 100 \\
\hline
\end{tabular}

Tabela 2. Quantidade de Teses e Dissertações distribuídas dos orientadores.

\begin{tabular}{|c|c|c|c|c|}
\hline Orientadores (Instituição) & Qtd. Diss. & Qtd. Teses & Total & Qtd. Instituição \\
\hline Kubo, O. M. (UFSC) & 4 & 2 & 6 & \multirow[t]{3}{*}{10} \\
\hline Botomé, S. P. (UFSC) & 1 & 1 & 3 & \\
\hline Medeiros, J. G. (UFSC) & 1 & 0 & 1 & \\
\hline Assis, F. R. P. (PUC-SP) & 2 & 0 & 2 & \multirow[t]{5}{*}{8} \\
\hline Gianfaldoni, M. H. T. A. (PUC-SP) & 2 & 0 & 2 & \\
\hline Luna, S. V. (PUC-SP) & 2 & 0 & 2 & \\
\hline Banaco, R. A. (PUC-SP) & 1 & 0 & 1 & \\
\hline Gioia, P. S. (PUC-SP) & 1 & 0 & 1 & \\
\hline Kienen, N. (UEL) & 1 & 0 & 1 & \multirow[t]{2}{*}{2} \\
\hline Marinho, M. L. (UEL) & 1 & 0 & 1 & \\
\hline Gimenes, L. S. (UNB) & 1 & 0 & 1 & \multirow[t]{3}{*}{3} \\
\hline Neto, J. M. O. C. (UNB) & 0 & 1 & 1 & \\
\hline Todorov, J. C. (UNB) & 0 & 1 & 1 & \\
\hline Assis, G. (UFPA) & 0 & 1 & 1 & \multirow[t]{2}{*}{2} \\
\hline Barros, R. S. (UFPA) & 1 & 0 & 1 & \\
\hline Bori, C. M. (USP) & 0 & 1 & 1 & 1 \\
\hline Total & 19 & 7 & 26 & 26 \\
\hline
\end{tabular}

Nota. Qtd. = Quantidade; Diss. = Dissertação. 
Foram identificados 16 orientadores que participaram da produção de dissertações e teses. Os orientadores com maior produção na área foram Olga Mitsue Kubo (UFSC), com seis trabalhos, Silvio Paulo Botomé (UFSC), com três trabalhos e, em seguida, Fátima R. P. Assis, Mônica H. T. A. Gianfaldoni e Sérgio Vasconcelos de Luna com dois trabalhos cada, sendo esses três últimos orientadores da PUC-SP. Os demais orientadores possuem uma produção cada - quando somadas essas dissertações e teses orientadas chega-se ao total de 11 estudos.

Quanto à produção por instituição, destacaram-se a Universidade Federal de Santa Catarina (UFSC), com dez trabalhos (38,46\%), e a Pontifícia Universidade Católica de São Paulo (PUC-SP), com oito trabalhos (30,76\%). A Universidade de Brasília (UNB) apresentou três trabalhos (11,53\%). Em seguida aparece a Universidade Estadual de Londrina (UEL) e a Universidade Federal do Pará (UFPA), ambas com dois trabalhos (7,69\%), e por fim, a
Universidade Federal de São Paulo (USP), com um trabalho (4\%). Observou-se, portanto, concentração da produção nacional em dois estados: Santa Catarina e São Paulo.

A Tabela 3 exibe a quantidade de autores que mais publicaram artigos. Foram considerados mais "produtivos" aqueles autores com pelo menos duas publicações. Pode-se observar que Botomé, da UFSC, se destaca com três publicações. Em seguida apareceram, Milani, da UNB, Borba, Costa e Ramos, que são da UFPA, e Miguel, da California State University, todos com duas publicações cada. Alguns desses autores possuem publicações em conjunto entre si e publicações com pesquisadores que foram incluídos na categoria outros. Justamente porque cada autor foi contabilizado individualmente é que o total de estudos foi superior a 19 (número total de artigos selecionados nesta pesquisa). Os 31 autores mencionados na Tabela 3 no conjunto "Outros" contam com uma publicação cada.

Tabela 3. Distribuição dos autores que mais publicaram artigos.

\begin{tabular}{llll}
\hline Autor & Instituição & Anos & Total \\
Botomé, S. P. & UFSC & $1981,2013,2012$ & 3 \\
Borba, A. & UFPA & 2014,2016 & 2 \\
Costa, T. D. & UFPA & 2014,2016 & 2 \\
Miguel, C. F. & California State University & 2001,2003 & 2 \\
Milani, I. & UNB & 1988,1989 & 2 \\
Ramos, C. C. & UFPA & 2014,2016 & 2 \\
Outros & --- & --- & 31 \\
\hline Total & & & 44 \\
\hline
\end{tabular}

\section{Discussão}

O objetivo desta revisão de literatura foi descrever quantitativamente, no período de 1970 a 2017, a produção científica brasileira relacionada à Análise do Comportamento nas Organizações (ACO). Verificou-se que o período de maior produção de dissertações e teses foi entre 2010 e 2011; no caso de artigos, destacou-se o ano de 2013. A produção de artigos foi menor que a de dissertações e teses, o que faz sentido porque uma publicação de artigo pode demorar muito, enquanto a publicação de uma dissertação ou tese depende da conclusão e defesa de um trabalho que precisa respeitar um prazo limite de, aproximadamente, dois a quatros anos, respectivamente. Além disso, muitos artigos são originados a partir de dissertações e teses. 
Os dados do presente estudo sugerem que há um número bem maior de dissertações (19) do que de teses (7). Foram levantadas algumas hipóteses para explicar o menor número de teses: (a) as pesquisas iniciadas no mestrado não tiveram continuidade no doutorado porque os pesquisadores optaram por mudar o seu tema de pesquisa ou não fizeram/iniciaram doutorado; (b) muitos programas de pós-graduação brasileiros ainda não possuem cursos de doutorado e nem todos têm orientadores analistas do comportamento que trabalhem com ACO; (c) o mercado de trabalho que contrata psicólogos analistas do comportamento para atuar em organizações, talvez valorize pouco o título de doutor. Esse dado sobre poucas teses e uma possível descontinuidade entre mestrado e doutorado sugere ser relevante ampliar e aprofundar as oportunidades de pesquisa em ACO, bem como a divulgação das pesquisas por meio da realização de eventos, publicação de livros, ampliação da biblioteca de OBM etc., conforme exame de Gusso e De Luca (2017).

Não se trata de defender aqui que todos precisem cursar um doutorado. Mas de indicar uma preocupação de que os pesquisadores, com a realização do doutorado, possam participar de programas de capacitação que lhes ofereçam tempo para realizar pesquisas mais robustas. Teses possuem um potencial maior de impacto científico. Logo, as repercussões para a prática profissional do psicólogo tendem a ser ampliadas. Isso é fundamental em um campo de atuação profissional no qual psicólogos parecem se orientar pouco por critérios científicos em suas intervenções, conforme revelam estudos de Gusso et al. (2019) e Pereira, Cobêro e Primi (2003).

Para além dessa questão sobre a entre quantidade de teses em relação à quantidade de dissertações, ou de artigos em relação a teses/dissertações, importa destacar que a produção de ACO parece pequena. Existe uma variável importante a considerar para explicar isso. Pode existir um entrave para pesquisas nas organizações e, especialmente, estudos com maior duração, como nos casos de doutorados. É possível que gestores, de um modo geral, e empresários, em particular, ainda possuam uma visão restrita sobre a atuação de psicólogos em organizações (Gusso et al., 2019), e uma percepção ainda mais restrita acerca da importância de pesquisas de psicólogos em organizações. Nesse último caso, talvez isso seja fruto de uma visão de senso comum da Psicologia como uma coleção de teorias sobre os seres humanos sem muita vinculação ou necessidade de exame científico (Ambridge, 2016).

Ao mesmo tempo, talvez não se trate de uma questão relacionada à Psicologia, mas com a atuação da Gestão de Pessoas. Muitas organizações adotam práticas de seleção de pessoas, por exemplo, que há muito tempo têm sido criticadas por sua baixa validade preditiva, como é o caso das entrevistas de emprego com questões abertas (Moore, 2017). Isso sugere uma baixa preocupação com pesquisas nessa área, o que pode ajudar a explicar que exista um número pequeno de pesquisas e pesquisadores.

Ao mesmo tempo, é preciso lembrar que pesquisas de psicólogos em organizações envolvem, geralmente, a coleta de dados com os trabalhadores. Nesse processo, pode ser necessário retirá-los de suas atividades, reduzir sua produtividade ou modificar o seu ambiente laboral, o que pode influenciar no seu desempenho. Desse modo, há um custo para a organização que pode ser considerado alto demais pelos gestores tendo por base o que se afirmou nos parágrafos anteriores.

Finalmente, é preciso lembrar que muitos psicólogos que atuam em organizações não estão, necessariamente, vinculados à academia. Talvez eles possuam a possibilidade de coletar dados ou compartilhar dados com pesquisadores. Contudo, existem implicações éticas a considerar. O psicólogo sem vinculação acadêmica não conseguiria uma pesquisa científica com humanos em contexto organizacional porque ele sequer poderia conseguir aprovação de um comitê de ética, além de outras implicações como o uso de Termos de Consentimento Livre e Esclarecido em processos seletivos e de avaliação de desempenho (Resolução CNS n. 466, 2012). Será que candidatos e trabalhadores, respectivamente, estariam consentindo participar de modo livre? Naturalmente, os procedimentos éticos são fundamentais e necessários. $\mathrm{O}$ destaque aqui é apenas no sentido de que menos pesquisas sobre ACO podem estar associadas a um custo de resposta que os próprios psicólogos podem considerar elevado. Além disso, a comunidade de analistas do comportamen- 
to atuando em organizações parece ser pequena, ao menos quando se consideram dados da Região Sul (Gusso et al., 2019), que concentra muitas universidades e programas de pós-graduação que formam esses profissionais.

Nas nuvens de palavras o termo comportamento destacou-se, o que indica a centralidade desse conceito nos estudos e na atuação do analista do comportamento em organizações, corroborando ponderações de Gusso e De Luca (2017) sobre o que caracteriza a atuação de um analista do comportamento em organizações. Outros termos como organizacional ou organizações, trabalho e profissional também apareceram com frequência. Chama a atenção, porém, termos que não aparecem e são considerados importantes na atuação do psicólogo em organizações, tais como "saúde" ou "saúde do trabalhador", ou precarização do trabalho (Zanelli et al., 2014). Também não apareceram de forma clara expressões relativas às subáreas de atuação de um psicólogo em organizações, tais como recrutamento e seleção, avaliação de desempenho, remuneração estratégica etc. (Zanelli et al., 2014). Isso parece indicar a necessidade de que analistas do comportamento ampliem o escopo dos seus estudos de modo a abarcar outros fenômenos relevantes do contexto organizacional (conforme sugerem Gusso et al., 2019), e/ou que incluam em seus trabalhos expressões que ajudem a promover aproximações com o conhecimento existente na Psicologia Organizacional e do Trabalho.

No que diz respeito aos objetivos dos estudos, verificou-se que os pesquisadores têm se concentrado em trabalhos de base com a função de orientar a atuação profissional, que envolvem a caracterização de comportamentos profissionais e o aperfeiçoamento ou esclarecimento de conceitos. Esse tipo de estudo é fundamental para uma atuação cientificamente orientada , ainda mais quando se considera que psicólogos têm conferido pouca atenção para atividades básicas como é o caso das análises de cargo que são essenciais para a realização de outros processos de RH (ver Gusso, 2015). Ao mesmo tempo, o estudo da eficiência de intervenções, que consiste em um aspecto importante para o desenvolvimento da área, permanece baixo. Esse é o tipo de trabalho que precisa ser conduzido para demonstrar a efetividade da atuação do analista do comportamento em organizações (Careli, 2013), o que, aliás, compõe a caracterização de uma intervenção analítico-comportamental em contexto aplicado (Baer, Wolf, \& Risley, 1968, 1987). O mesmo acontece em relação ao desenvolvimento de instrumentos, que, segundo Rocha (2016), podem servir como um suporte importante na atuação profissional.

Uma explicação para esse dado é que talvez o número de pesquisadores de $\mathrm{ACO}$ ainda seja pequeno e a demanda por pesquisas mais básicas ainda seja grande para consolidar a área. Ao mesmo tempo, com as possíveis dificuldades de condução de pesquisas em ambientes organizacionais já apontadas, torna-se mais provável a realização de estudos nos quais o pesquisador depende menos da colaboração de uma empresa para conduzir a sua investigação.

Os dados do presente estudo indicam, ainda, que existe uma concentração da produção nacional nas regiões sul e sudeste do país, especificamente, nos estados de Santa Catarina (UFSC) e São Paulo (PUC-SP), respectivamente. Algumas estratégias, porém, podem estimular o aumento da pesquisa sobre ACO em outros estados, tais como a realização de eventos científicos online (e.g., Jornada de Análise do Comportamento do Brasil), além da produção de livros que possam apresentar conceitos e propostas de intervenção em uma linguagem mais acessível para uma comunidade mais ampla de psicólogos.

Foi possível identificar, ainda, os professores que mais orientaram na produção de teses e dissertações. Os orientadores com maior produção na área foram Olga Mitsue Kubo (com seis trabalhos) e Silvio Paulo Botomé (com três trabalhos). Esses professores tiveram suas aposentadorias concedidas em 2013 (Gusso \& De Luca, 2017), sendo necessário verificar em estudos futuros novos orientadores que poderão destacar-se nesse campo de pesquisa e atuação profissional em Psicologia. Na produção de artigos foi o professor Sílvio Paulo Botomé o autor que mais se destacou. Isso é compreensível, pois se os pós-graduandos são incentivados a publicar as suas teses e o professor Sílvio foi um dos que mais orientou, então tendia a destacar-se também na publicação de artigos.

Este estudo possui limitações que precisam ser consideradas para orientar pesquisas futuras. Um 
aspecto importante a destacar é que não foi caracterizada a totalidade dos trabalhos em ACO referente ao período delimitado - não foram considerados, por exemplo, trabalhos apresentados em eventos, livros e capítulos de livro. Além disso, foram utilizados poucos descritores e que não deram conta de identificar estudos que utilizaram diferentes terminologias. Em relação ao critério de ser ou não um estudo de Análise do Comportamento, sabe-se que essa é uma questão problemática porque o relato do autor não consiste em uma fonte inequívoca para classificar uma pesquisa como analítico-comportamental. Contudo, foi um critério útil para viabilizar este estudo exploratório. A partir do banco de dados criado e das contribuições apresentadas, estudos futuros poderão desenvolver critérios mais rigorosos para orientar novas revisões de literatura sobre ACO.

Para as próximas pesquisas, sugere-se: (a) aperfeiçoar os descritores para lidar com a diversidade terminológica da área; (b) incluir o levantamento de capítulos de livros e apresentações de trabalhos em congressos (tais como os Encontros Anuais da Associação Brasileira de Psicologia e Medicina Comportamental e as Reuniões da Sociedade Brasileira de Psicologia); (c) partir das pesquisas divulgadas na Biblioteca de OBM; (d) consultar referências descritas em cada pesquisa recuperada e selecionada; e, por fim, (e) propor uma definição que permita delimitar com clareza o que deve ser considerado como ACO. Com este estudo pretendeu-se quantificar, divulgar e estimular a produção sobre ACO. Avalia-se que assim será possível auxiliar no desenvolvimento dessa subárea da Análise do Comportamento que possui elevado potencial contributivo para as organizações.

\section{Referências}

Ambridge, B. (2016). Psi-q. Rio de Janeiro: Sextante. Aureliano, L., Arima, C. Y., \& Careli, G. G. (2013). Análise do comportamento e trabalho: Análise do Comportamento Aplicada às Organizações. Boletim Paradigma, 37-40. Recuperado de: http://bit.ly/2G7jeCc

Austin, J. (2000). Performance analysis and performance diagnostics. Em: J. Austin \& J. E. Carr (Eds.), Handbook of Applied Behavior Analysis (pp. 321-349). Reno, Nevada: Context Press.

Baer, D. M., Wolf, M. M., \& Risley, T. R. (1968). Some current dimensions of applied behavior analysis. Journal of Applied Behavior Analysis, 1(1), 91-97. doi: 10.1901/jaba.1968.1-91

Baer, D. M., Wolf, M. M., \& Risley, T. R. (1987). Some still current dimensions of applied behavior analysis. Journal of Applied Behavior Analysis, 20(4), 313-327. doi: 10.1901/ jaba.1987.20-313

Brasil. Conselho Nacional de Saúde. Resolução 466/2012. Trata de pesquisas em seres humanos e atualiza a resolução 196. [Internet]. Diário Oficial da União. Recuperado de: http://conselho.saude.gov.br/resolucoes/2012/Reso466.pdf

Borba, A., Ramos, C. C., \& Costa, T. D. (2017). O surgimento da análise do comportamento aplicada às organizações. Em D. L. O. V. Boas, F. Cassas, H. L. Gusso, Comportamento em Foco vol. 5 (pp. 13-27). São Paulo: Associação Brasileira de Psicologia e Medicina Comportamental - ABPMC. Recuperado de: http://bit.ly/2QljZvZ

Careli, G. G. (2010). Administração por quem e para quem: uma análise behaviorista de processos de recursos humanos. (Trabalho de Conclusão de Curso, Curso de Psicologia, Pontifícia Universidade Católica de São Paulo). Recuperado de: http://bit.ly/2zKuaju

Careli, G. G. (2013). OBM Funciona? Uma meta-análise de artigos publicados na área. (Dissertação de Mestrado, Programa de PósGraduação em Psicologia Experimental: Análise do Comportamento, Pontifícia Universidade Católica de São Paulo). Recuperado de: http:// bit.ly/2Ed4tvd

Colepicolo, E. (2014). Buscando informação científica de qualidade para pesquisa em Psicologia. Estudos Interdisciplinares em Psicologia, 5, 2, 133-142. doi: 10.5433/2236-6407.2014v5n2p133

Daniels, A. C., \& Bailey, J. E. (2014). Performance management: Changing behavior that drives organizational effectiveness. Aubrey Daniels Internacional, Inc.: Atlanta, GA.

Gusso, H. L. (2015). Análise de cargo, recrutamento e seleção: manual prático para aumentar a eficácia na contratação de profissionais. Curitiba: N1 Tecnologia Comportamental. 
Gusso, H. L. (2017). Desafios ao Analista do Comportamento no Campo Organizacional Brasileiro. Em D. L. O. V. Boas, F. Cassas, H. L. Gusso, Comportamento em Foco vol. 5 (pp. 77-86). São Paulo: Associação Brasileira de Psicologia e Medicina Comportamental ABPMC. Recuperado de: http://bit.ly/2QljZvZ

Gusso, H. L., \& De Luca, G. G. (2017). Organizações como Sistemas Comportamentais: Considerações para a Delimitação do Campo de Atuação. Em D. L. O. V. Boas, F. Cassas, H. L. Gusso, Comportamento em Foco vol. 5 (pp. 28-40). São Paulo: Associação Brasileira de Psicologia e Medicina Comportamental ABPMC. Recuperado de: http://bit.ly/2QljZvZ

Gusso, H. L., Alvarenga, A. da S., Nunes, P. P., Nunes, M. F. O., De Luca, G. G., \& Oliveira, M. Z. de. (2019). Psicologia Organizacional e do Trabalho no Sul do Brasil: características dos profissionais, da atuação e dos contextos de trabalho. Revista Psicologia Organizações e Trabalho, 19(3), 644-652. doi: 10.17652/ rpot/2019.3.16131

Henklain, M. H. O., Carmo, J. D. S., \& Haydu, V. B. (2017). Produção analítico-comportamental brasileira sobre comportamento matemático e de ensinar matemática: dados de 1970 a 2015. Temas em Psicologia, 25(3), 1453-1466. doi: 10.9788/TP2017.3-24

Kienen, N., \& Wolf, S. (2002). Administrar comportamento humano em contextos organizacionais. Revista Psicologia: organizações e trabalho, 2(2), 11-37. Recuperado de: http://bit.ly/2BZtacQ

Miguel, C. F. (2001). Uma introdução ao gerenciamento comportamental de organizações. Em M. Delitti (Org.), Sobre comportamento e cognição (pp. 265-274). São Paulo: ESETec.

Moore, D. A. (2017). How to improve the accura$c y$ and reduce the cost of personnel selection. California Management Review, 60(1), 8-17. doi: $10.1177 / 0008125617725288$

Moreira, E. G. (2005). O Journal of Organizational Behavior Management como um veículo para a publicação de pesquisa em análise aplicada do comportamento, na área de organizational behavior management. (Dissertação de Mestrado, Programa pós-graduação em Psicologia Experimental: Análise do Comportamento,
Pontifícia Universidade Católica de São Paulo). Recuperado de: http://bit.ly/2UsjrDe

Moreira, W. (2004). Revisão de literatura e desenvolvimento científico: Conceitos e estratégias para confecção. Janus, 1(1), 19-30. Recuperado de http://bit.ly/2rq64pF

Pereira, F. M., Primi, R., \& Cobêro, C. (2003). Validade dos testes utilizados em seleção de pessoal segundo recrutadores. Psicologia: Teoria e Prática, 5(2), 83-98. Recuperado de https://bit. ly/2Y3VKEr

Ratinaud, P. (2009). IRAMUTEQ: Interface de R pour les Analyses Multidimensionnelles de Textes et de Questionnaires [Computer software]. Recuperado de: http://www.iramuteq. org

Rocha, G. G. (2016). Performance Diagnostic Checklist - Human Services: Adaptação e aplicação em uma empresa brasileira (Dissertação de mestrado, Programa de Estudos Pósgraduados em Psicologia Experimental: Análise do Comportamento, Pontifícia Universidade Católica de São Paulo). Recuperado de: https:// goo.gl/bjhJvH

Todorov, J. C., \& Hanna, E. S. (2010). Análise do comportamento no Brasil. Psicologia: teoria e pesquisa, 26(25ANOS), 143-154. doi: http:// dx.doi.org/10.1590/S0102-37722010000500013 Zanelli, J. C., Bastos, A. V. B., \& Rodrigues, A. C. A. (2014). Campo profissional do psicólogo em organizações e no trabalho. In: J. C. Zanelli; J. E. Borges-Andrade; A. V. B. Bastos (Orgs.), Psicologia, Organizações e Trabalho (pp. 549582). Porto Alegre: Artmed.

\section{Informações do Artigo}

\section{Histórico do artigo:}

Submetido em: 22/12/2018

Primeira decisão editorial: 09/07/2020

Aceito em: 02/09/2020

Editor associado: Thiago Dias Costa 